\title{
Sonographic Appearance of Synovial Crystal Deposits in 2 Patients with Gout
}

ANGEL CHECA, MD, Division of Rheumatology, Drexel University College of Medicine, 219 North Broad St., Philadelphia, Pennsylvania 19107, USA. Address correspondence to Dr. Checa; E-mail: checaag@ comcast.net. J Rheumatol 2012;39:2362-3; doi:10.3899/jrheum.120513

The identification of monosodium urate (MSU) deposits within the synovium in gout is well known by arthroscopic and pathologic descriptions ${ }^{1,2}$. However, this has not been systematically studied by ultrasound (US). Most studies about US and crystal-related arthritis have focused on the appearance and location of the crystal aggregates either within or on the surface of the cartilage. In gout, deposits appear as a hyperechoic enhancement on the surface of the articular cartilage called the "double contour" sign 3 .

Two patients with gout that disclosed MSU crystals in the synovial fluid and synovium biopsies are presented. A characteristic "double contour" sign and deposits on the synovium were identified by US.

The first patient, a 47-year-old male, underwent arthroscopy because of a hypertrophic mediopatellar plica. A sonographic study performed 1 week earlier revealed several bright hyperechoic dots within the synovial effusion (Figure 1a). The arthroscopy also showed tophaceous material free-floating within the fluid (Figure 1b).

The second patient, a 51-year-old male, underwent arthroscopy for a meniscal injury. The imaging showed diffuse granular tophaceous deposits in the synovium (Figure 2a). In a sonographic study performed 2 weeks later, multiple nonshadowing hyperechoic dots in the synovium were observed at the lateral recess (Figure 2b).
Although this sonographic image of the synovium is not specific for gout ${ }^{4,5}$, it reinforces the presence of the "double contour" sign, particularly in any circumstances in which arthrocentesis is not an option. The presence of synovial deposits warrants a sonographic examination, including examination of other joints, looking for cartilage aggregates. Future studies about sonomorphologic aspects of the synovium in crystal-induced arthritis are needed.

\section{REFERENCES}

1. Checa A, O'Connor CR. Gout attack and stiff knee in an airline passenger in economy class. Arthritis Rheum 2009;60:1361.

2. Schumacher HR. Pathology of the synovial membrane in gout. Light and electron microscopic studies. Interpretation of crystals in electron micrographs. Arthritis Rheum 1975;18:771-82.

3. Filippucci E, Scirè CA, Delle Sedie A, Iagnocco A, Riente L, Meenagh G, et al. Ultrasound imaging for the rheumatologist. Sonographic assessment of the knee in patients with gout and calcium pyrophosphate deposition disease. Clin Exp Rheumatol 2010;28:2-5.

4. Grassi W, Meenagh G, Pascual E, Filippucci E. 'Crystal clear' - Sonographic assessment of gout and calcium pyrophosphate deposition diseases. Sem Arthritis Rheum 2006;36:197-202.

5. Wakefield R, O'Connor P. All that glimmers is not gold. Sem Arthritis Rheum 2007;37:133-4.
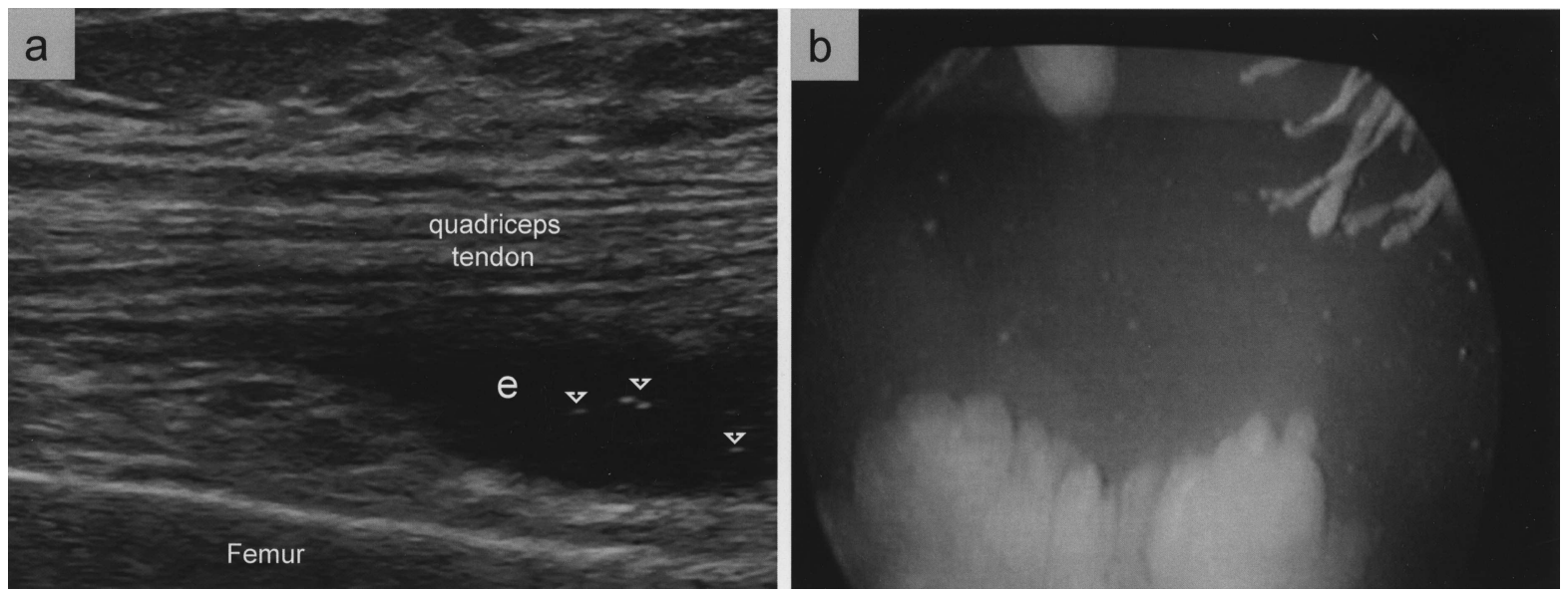

Figure 1. Panel a. Longitudinal sonographic view of the suprapatellar pouch showing several bright hyperechoic dots, snowstorm-like appearance (arrowheads), within synovial effusion (e). Panel b. Tophaceous material released, giving the appearance of "snowball" in an arthroscopic view of the suprapatellar recess. 

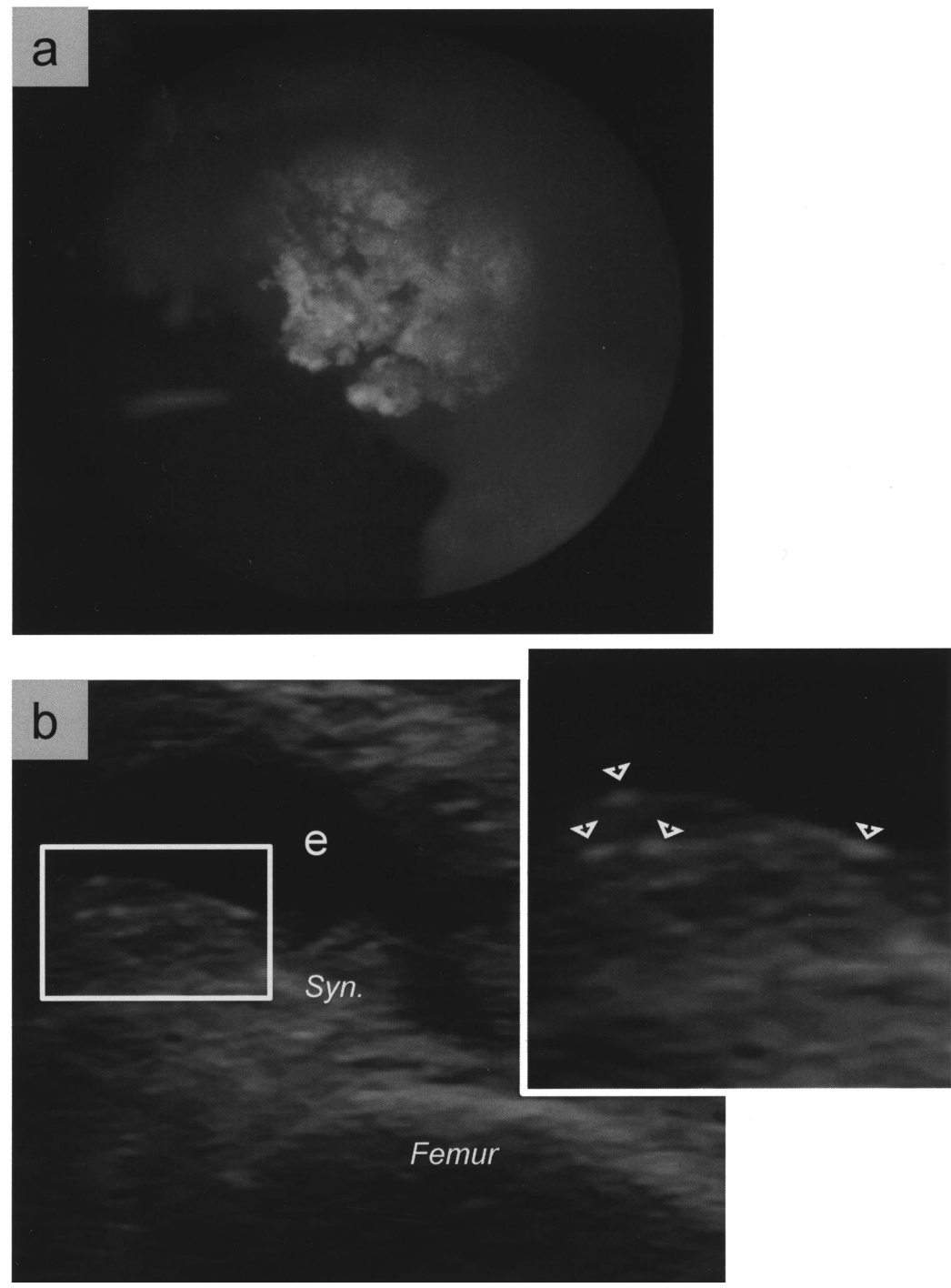

Figure 2. Panel a. An arthroscopic view of the lateral recess showing a gout tophus in the hyperemic synovium. Panel b. Transverse sonographic view of the same area shows nonshadowing hyperechoic dots within a hypertrophic synovium (Syn.), surrounded by a moderate amount of fluid (e). 